\title{
"Inverted Microcarriers" for Cell Cultures Made by Polymerization of Shells around Agarose Microspheres in a Non-Cytotoxic Procedure
}

\author{
Ch. Cadic, Ch. Baquey, and B. Dupur* \\ Inserm U.306-146, rue Léo Saignat-33076 Bordeaux Cedex (France) \\ *Inserm U.306-Université de Bordeaux II, 146, rue Léo Saignat, \\ 33076 Bordeaux-Cédex (France)
}

(Received November 27, 1990)

\begin{abstract}
A new process for microencapsulating cells is described. Cells in solution in agarose were extruded from a syringe into a paraffin oil containing medium. Solid microspheres were produced when the agarose gelled. A rigid interface around each bead was produced by polymerization of a latex seeded solution of a mixture of $5 \%$ acrylamide and $0.25 \%$ bisacrylamide. Cell viability was demonstrated by radioimmunoassay of prolactin liberated from encapsulated pituitary cells.

KEY WORDS Microencapsulation / Microcarriers / Cell Culture / Seeded Polymerization /
\end{abstract}

Large numbers of cultured cells are required for numerous purposes including: virus production, preparation of large quantities of membranes, production of interferon, etc. In order to increase the surface area for cell attachment, bead-like particles can be added to the culture vessel. However, this method is restricted to anchorage-dependent cells. Furthermore, cells growing on the surface of solid beads (microcarriers) are exposed, and may be damaged by collision with other beads, the impeller blades or the walls of the vessel. It has been suggested that cell culture could be carried out within microbeads, but in this case, the procedures for making microbeads must not be cytotoxic as living cells are present throughout the whole procedure. Until now, only ionic or insoluble polymers have been employed. ${ }^{1-3}$

We describe the preparation of microbeads in a two step process: living cells are embedded in microspheres of agarose, and then a polymer shell is formed round each bead in a latex seeded polymerization process. This gives rise to a three dimensional network of covalently bound molecules which produces an artificial membrane with more permanent properties. We tested the toxicity of the procedure by radioimmunoassay of the secretion of prolactin from pituitary cells either alone, embedded in agrarose or microencapsulated by our method.

\section{MATERIALS AND METHODS}

\section{Preparation of Cells}

Pituitary cells of lactating female rats were isolated by sequential enzymatic digestion: trypsin, DNase, EDTA, as described by Hopkins. ${ }^{4}$ The fragments were dispersed into single cells by gentle suction and extrusion through a Pasteur pipette. The cell suspension was then sedimented through a layer of new-born calf serum. A fraction was taken for a cell count in a hemocytometer, and viability was assessed by the trypan blue exclusion test. $5 \cdot 10^{6}$ cells were suspended in $10 \mathrm{ml}$ of Dulbecco modified minimum essential medium DMEM (GIBCO), supplemented with $10 \%$ new-born 
calf serum and $50 \mu \mathrm{g} \mathrm{ml}^{-1}$ gentamycin, placed in roller tubes at $37^{\circ} \mathrm{C}$ for a least three days before encapsulation.

\section{Latex}

Polyacrylamide latex were synthesized from an acrylamide bisacrylamide mixture (Biorad) dispersed in a microemulsion. The oil was a narrow isoparaffinic mixture (Isopar M; Esso Chemie), which was filtered before use (boiling range $207-257^{\circ} \mathrm{C}$ ). The emulsifiers were a blend of sesquioleate sorbitan (ARLACEL, HLB $=3.7$ ) and a polyoxyethylene sorbitol hexaoleate with 40 ethylene oxide residues $(G$ 1086, HLB $=10.2$ ) supplied by Atlas Chemical Industrie N.V. and used without purification. Lithium chloride was from Prolabo, TEMED: $N, N, N^{\prime}, N^{\prime}$-tetramethylethylenediamine riboflavin (Sigma) and AIBN: azobisisobutyronitrile (Merck) were kept at $4^{\circ} \mathrm{C}$ in the dark.

Microemulsions were prepared $(\mathrm{w} / \mathrm{w})$ by addition under stirring of the aqueous phase (water + acrylamide/bisacrylamide + electrolyte) to the oil phase (blend of Isopar $\mathrm{M}+$ surfactants). When used, riboflavin was in the aqueous phase and AIBN in the oil phase. Microemulsions were degassed under vacuum to remove oxygen prior to polymerization. The light source was a mercury lamp placed in a water-jacketed reaction vessel. The temperature of the polymerization reaction was regulated at $25^{\circ} \mathrm{C}$. After polymerization, polymers were recovered by precipitation in an excess of a methanol/acetone mixture, followed by several washings. The polymers were filtered and then freeze-dried to prevent imidization of amide groups. The extent of polymerization and purity of the polymers were analyzed by IR spectroscopy. The size of the unprecipitated latex particles was determined by quasi-elastic light scattering QELS: microemulsions were diluted with Isopar to give a volumic fraction of the dispersed phase between 0.5 and $2 \%$. Measurements were also performed after dispersion of the microemulsion in $30 \%$ acrylamide solution.
Polystyrene latex (LB-1 type, $100 \mathrm{~nm}$ diam.) was purchased from Sigma.

\section{Microcapsules}

The first embedding solution was prepared with agarose, an almost neutral polysaccharide. A particular feature of the gelling of agarose is the marked temperature difference between the gel-sol and the sol-gel transition. We used a type (Sigma type IX) that gels around $15^{\circ} \mathrm{C}$ in a $1 \%$ solution in order to be able to mix with living cells before solidification of the gel. The microbeads were produced from an arrangement of two coaxial tubes: the living cells $\left(5.10^{+6}\right)$ in suspension in the agarose solution $(0.5 \mathrm{ml})$ were extruded from the needle of a syringe ( $1 \mathrm{ml}$ insulin syringe, $26 \mathrm{G} \times 1 / 2$ " needle. Pharma plast. W. Germany) inside a slightly larger polyethylene capillary tube through which paraffin oil was pumped $(0.56 \mathrm{~mm}$ inner diameter, Biotrol Pharma, France). At the end of the capillary, the beads were cooled to $4^{\circ} \mathrm{C}$ in a paraffin oil bath to ensure rapid gelling. The beads were then subjected to a second embedding procedure. With the aid of a dissecting microscope, the polymerizable aqueous solution was placed at the interfaces between each bead and the oil medium using a time and pressure adjustable pneumatic device (Microelectronic system, EFD France). The polymerizable solution surrounds the beads in the oil medium. The shells were hardened by a photochemical process. The polymerizable solution was a mixture of monomers ( $5 \%$ acrylamide, $0.25 \%$ bisacrylamide) containing a suspension of about $10^{13}$ latex particles/ml (polystyrene or polyacrylamide latex). In each case, $10^{-5} \mathrm{M}$ riboflavin as sensitizer and $4.10^{-4} \mathrm{M}$ TEMED as catalyst were added. Just before use, TEMED was diluted to $1 \%$ in HEPES $\left(N\right.$-2-hydroxyethylpiperazine- $N^{\prime}$-ethanesulfonic acid) buffer to keep the $\mathrm{pH}$ of the solution at 7.8 .

Microspheres surrounded by the polymerizable solution were removed from the bath by 


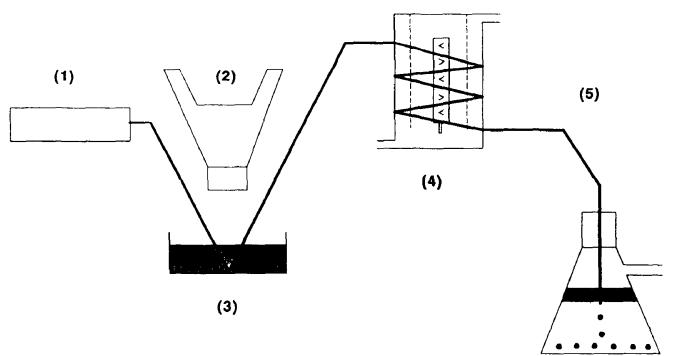

Figure 1. Schema of the polymerization set-up: (1) pneumatic device for polymerizable solution, (2) binocular microscope, (3) petri dish containing agarose embedded cells in oil, (4) cooled irradiating vessel, (5) receiving flask.

aspiration into a polyethylene capillary tube. The extension of the capillary was coiled round a transparent glass column cooled with water and containing a high pressure mercury lamp. Light initiated the photochemical reaction, and polymerization took place during the transit of the beads along the capillary. The capillary ended in a flask connected to a vacuum pump which kept the oil phase flowing. The flask contained culture medium, and microcapsules coming into the flask dropped to the bottom while the oil phase stayed at the top (Figure 1).

To estimate cytotoxicity, secretion of prolactin from pituitary cells was measured during the different steps of the procedure. Samples of cells $\left(10^{6}\right.$ cells embedded in $10^{3}$ microcapsules) were introduced into the incubation chambers which were constructed from the bodies of syringes with the ends covered with nylon mesh. Cells were equilibrated at $37^{\circ} \mathrm{C}$ for at least $90 \mathrm{~min}$ in Krebs Ringer bicarbonate solution before the tests were run. Three $45 \mathrm{~min}$ incubation periods were performed with the incubation medium both with and without $50 \mathrm{mM} \mathrm{KCl}$. At the end of each incubation time, media were removed and stored at $-25^{\circ} \mathrm{C}$ until assay.

\section{RESULTS}

In order to polymerize latex inside microemulsions, we employed the concentrations described by Candau. ${ }^{5}$ The emulsifier mixture
G 1086 + ARLACEL 83 was used to obtain a hydrophile/lipophile balance (HLB) of 9.3. Concentrations of emulsifier blend as low as $15 \%(\mathrm{w} / \mathrm{w})$ could be achieved by addition of $1.5 \mathrm{M}$ lithium chloride. The microemulsion domain, characterized by an homogeneous phase observed between crossed polarizers, was increased by addition of lithium chloride and acrylamide. Irradiation of this microemulsion did not alter the macroscopic physical characteristics of the mixture, which stayed clear with no change in viscosity. However, if the microemulsions were solubilized in acetone before irradiation, they produced a white precipitate after irradiation. Infrared spectra of Isopar, the emulsifier blend, and the precipitate after washing and purification demonstrated a high degree of polymerization and satisfactory purity. In the $1800-1600$ $\mathrm{cm}^{-1}$ region of the polymer spectra, the two broad absorption bands attributed to vibrations of $\mathrm{CO}-\mathrm{NH}_{2}$ groups, and referred to as the amide I and amide II bands were clearly observed. Washing in acetone and methanol eliminated excess Isopar and emulsifiers. The hydrodynamic diameter estimated from photon correlation spectroscopy was $85 \pm 5 \mathrm{~nm}$ in Isopar. This increased to $320 \mathrm{~nm}$ after dispersion in acrylamide solution. Segmentation of agarose in the hydrophobic medium resulted in microspheres whose diameter depended on the size of the capillary. With our system, the mean size of the beads was around 400 micrometers. Microspheres encapsulated in a polyacrylamide and/or polystyrene shell could be seen by examination under a dissecting microscope with orthogonal illumination; the first were translucid and the second appeared white, their size varied from 500 to 600 micrometers. The presence of the shells was also checked by raising the temperature above the melting point of agarose, although this killed the cells. The beads without a polymer shell dissolved completely in the medium.

Prolactin secretion from pituitary cells cultured for three days in suspension varied 


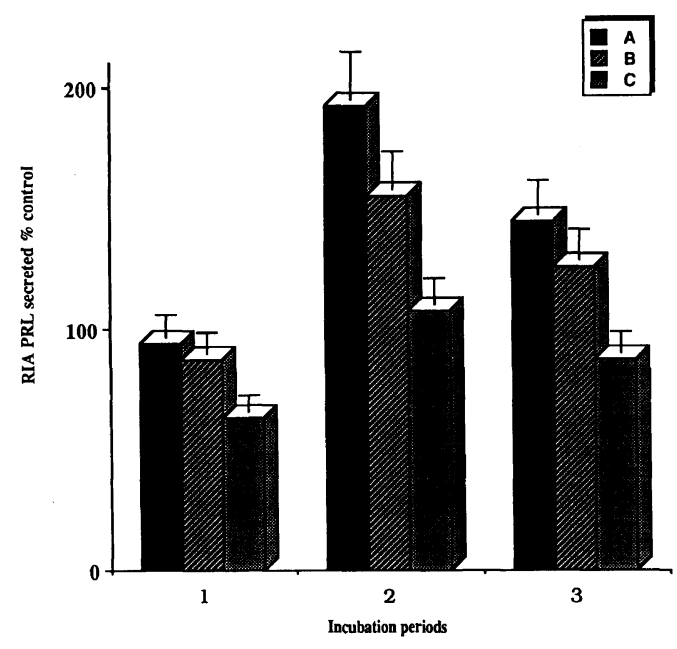

Figure 2. Prolactin secretion from pituitary cells for three consecutive 45 minutes incubations in basal $(1,3)$ or $50 \mathrm{mM}$ $\mathrm{KCl} \mathrm{KRB}$ solutions (2). Comparisons were made between cells from the same dissociation. Values are expressed as percentage of the secretion of the same number of non encapsulated cells (A); cells embedded in agarose (B); cells microencapsulated with latex supplemented polymerizable solution (C).

with the individual preparation ( 5 to $25 \mathrm{ng} /$ $\min / 10^{6}$ cells). We thus compared secretion from cells isolated on the same day. Some agarose beads containing pituitary cells were used as a control without asecond embedding. Embedding in agarose reduced basal and stimulated secretion by around $10 \%$. We found that $65 \%$ of the cellular activity was preserved after microencapsulation with the supplemented microlatex polymerizable solution. Adding $\mathrm{KCl}$ in the incubation medium resulted in increased secretions. Basal level recoveries were not obtained during the third incubation periods (Figure 2).

\section{DISCUSSION}

Agarose gel is a well-known matrix for cell cultures. ${ }^{6}$ Two different methods have been used for biocompatible embedding of cells in beads: the emulsion procedure, or extrusion facilitated by a coaxial air stream. Our approach attempted to combine the advantages of both processes, and spherical microcapsules were formed instantaneously with a relatively homogeneous size. Polymeric shells were made by polymerization of a latex suspension. This procedure was analogous to the seeded polymerization process used to produce structured latex. ${ }^{7}$ In seeded polymerization affinity of the monomers for the latex beads plays a prime role. ${ }^{8}$ Although the various steps of these polymerization reactions have not been completely worked out, polymerization does take place around each latex bead whose size increases progressively. ${ }^{9}$ As polyacrylamide latex are hydrophilic and polystyrene latex are hydrophobic, two different mechanisms were respectively account for the growth of acrylamide and polystyrene latex. In our experiments, mechanical forces induced by the transit of agarose beads through the capillary modified the purely chemical reaction, by approximating the latex particles, sticking them to each other and to the agarose beads, thereby facilitating formation of the polymer shell. In solutions without latex, higher monomer concentrations $(30 \% \mathrm{w} / \mathrm{w})$ had to be used to obtain a rigid capsule.

The continued secretion of prolactin demonstrated the lack of cytotoxicity of the process. The $\mathrm{KCl}$ induced hormonal secretion indicated that pituitary cells were yet responsive to alterations in Potassium levels. High values measured during the third incubation periods could be due to a gradual recovery to basal secretion as it was already observed with these cells. ${ }^{10}$ To our knowledge, this is the first description of a non toxic in situ polymerization for encapsulating living cells.

The permeability of the polymer shell depends on the choice of latex and monomer concentration. This can be adjusted to that the desired product either accumulated within the microcapsule or diffuses into the culture medium. 


\section{REFERENCES}

1. M. F. A. Goosen, G. M. O'Shea, H. M. Gharapetian, S. Chow, and A. M. Sum, Biothcnol. and Bioengineer, XXVII, 146 (1985).

2. K. Braun, B. Kuttler, H. Jahr, and H. J. Hahn, Horm. Metabol. Res., 19, 345 (1987).

3. R. M. Dawson, R. L. Broughton, W. T. K. Stevenson, and M. V. Sefton, Biomaterials, 8, 360 (1987).

4. C. R. Hopkins and M. G. Farquhar, J. Cell. Biol., 59, 276 (1975).

5. F. Candau, Z. Zekhnini, and J. P. Durand, J. Colloid Interf. Sci., 114, 398 (1986).
6. K. Nilsson, W. Scheirer, O. W. Merten, L. Ostberg, E. Liehl, H. W. D. Katinger, and K. Mosback, Nature, 302, 629 (1983).

7. W. D. Hergeth, H. J. Bittrich, F. Eichhorn, S. Schlenker, K. Schmutzzler, and U. J. Steinau, Polymer, 60, 1913 (1989).

8. J. C. Daniel, Makromol. Chem., Suppl., 10/11, 359 (1985).

9. J. C. De la Cal, R. Urzay, A. Zamora, J. Forcada, J. M. Asua, J. Polym. Sci. Polym. Chem., 28, 1011 (1990).

10. R. Drouhault, N. Abrous, J. P. David, and B. Dufy, Neuroendocrinology, 46, 360 (1987). 